() П.О. Румянцев*, В.А. Саенко, Д.С. Дзейтова, А.А. Трухин, М.С. Шеремета, К.Ю. Слащук, М.В. Дегтярев, С.С. Серженко, В.С. Ясюченя, С.М. Захарова, Я.И. Сирота

Национальный медицинский исследовательский центр эндокринологии, Москва, Россия

ОБОСНОВАНИЕ. Недостаточный клинический опыт проведения радиойодтерапии (РЙТ) болезни Грейвса (БГ) у детей и подростков в мировой практике и ограниченное понимание предикторов эффективности лечения.

ЦЕЛЬ. Изучить и выявить наиболее значимые предикторы эффективности РЙТ БГ в педиатрической группе пациентов.

МАТЕРИАЛЫ И МЕТОДЫ. В исследование включены 55 пациентов (Ж - 48, М - 7) в возрасте от 8 до 18 лет, получавших РЙТ по поводу БГ первично. Планирование РЙТ осуществлялось методом дозиметрического подхода. Анализируемые параметры включали пол, возраст, объем щитовидной железы (ЩЖ) по данным ультразвукового исследования до и через 6 месяцев после лечения, статус эндокринной офтальмопатии, длительность приема тиреостатиков, рецидив тиреотоксикоза на тиреостатической терапии в анамнезе, уровни свободного Т3 (свТ3), свободного Т4 (свТ4) и тиреотропного гормона (ТТГ) исходно и на 1, 3, 6 месяцы после лечения, антитела к рецептору ТТГ исходно и на 3 и 6 месяцы после лечения, индекс тиреоидного захвата ${ }^{99 m}$ Тс-пертехнетата на 10-20 минуте (\%), максимальный тиреоидный захват ${ }^{131}$ I (\%), удельный захват ${ }^{131}$ І (МБк/г) и назначенную активность ${ }^{131}$ І (МБк). Для статистических расчетов использованы точный тест Фишера, непараметрический тест Манна-Уитни, тест знаковых рангов Уилкоксона, логистические регрессионные модели, ROC-анализ, регрессия Кокса, метод Каплана-Майера, логарифмический ранговый тест.

РЕзУЛЬтАТЫ. Через 6 месяцев после РЙТ гипотиреоз развился у 45 (81,8\%) пациентов, эутиреоз - у 2 (3,6\%), и у $8(14,6 \%)$ сохранился тиреотоксикоз. В одномерном статистическом анализе меньший объем ЩЖ, более высокий уровень свТ4, низкий уровень антител к рецептору ТТГ, меньший индекс захвата 99mТс-пертехнетата и повышенный удельный захват ${ }^{131}$ I оказались связанными с достижением гипотиреоза. В многомерном логит-регрессионном анализе независимыми предикторами эффективности РЙТ были старший возраст пациента ( $p=0,011)$, меньший объем ЩЖ $(p=0,003)$ и более высокий уровень свТ4 ( $p=0,024)$. Единственным статистически значимым предиктором результата лечения во времени оказался исходный объем ЩЖ $(\mathrm{p}=0,011)$.

ЗАКЛЮЧЕНИЕ. Эффективность дозиметрического подхода при планировании РЙТ у детей и подростков с БГ составила 81,8\% через 6 месяцев. Независимыми предикторами эффективности РЙт были старший возраст пациента, меньший объем ЩЖ и более высокий уровень свТ4. Исходный меньший объем щЖ также был предиктором положительного результата лечения во времени. Представленные статистические модели могут быть использованы на практике для проспективной оценки вероятности эффективности РЙТ БГ у пациентов детскоподросткового возраста.

КЛЮЧЕВЫЕ СЛОВА: радиойодтерапия; дети и подростки; болезнь Грейвса; дозиметрическое планирование; ядерная медицина; тераностика.

\title{
PREDICTORS OF THE EFFICACY OF RADIOIODINE THERAPY OF GRAVES' DISEASE IN CHILDREN AND ADOLESCENTS
}

(c) Pavel O. Rumyantsev, Vladimir A. Saenko, Dali S. Dzeytova, Alexey A. Trukhin, Marina S. Sheremeta, Mikhail V. Degtyarev, Konstantin Y. Slashchuk, Sergey S. Serzhenko, Valentina S. Yasuchenia, Svetlana M. Zakharova, Yaroslav I. Sirota

Endocrinology Research Centre, Moscow, Russia

RATIONALE: Insufficient world-wide clinical experience in radioiodine therapy (RIT) for Graves' disease (GD) in children and adolescents, and limited knowledge of the predictors of RIT efficacy.

AIMS: Analysis and identification of the most significant predictors of the efficacy of RIT in children and adolescents with Graves' disease.

MATERIALS AND METHODS: A total of 55 patients (48 females and 7 males) aged from 8 to 18 years receiving primary RIT for GD were enrolled. RIT planning was based on the dosimetric method. Analyzed parameters included gender, age, ultrasound thyroid volume before and 6 months after treatment, the presence of endocrine ophthalmopathy, duration of 
antithyroid drug (ATD) therapy, relapse of thyrotoxicosis after ATD dose reduction, blood fT3, fT4 and TSH levels initially and at 1,3, 6 months after treatment, TSH receptor Ab initially and at 3 and 6 months after treatment, thyroid ${ }^{99 \mathrm{mTC}}$-pertechnetate uptake at 10-20 minutes (\%), maximum thyroid ${ }^{131} \mid$ uptake (\%), specific ${ }^{131} \mid$ uptake $(\mathrm{MBq} / \mathrm{g})$ and therapeutic ${ }^{131} \mathrm{I}$ activity $(\mathrm{MBq})$. Fisher exact test, non-parametric Mann-Whitney test, Wilcoxon signed-rank test, logistic regression modelling, ROC-analysis, proportional hazard model (the Cox regression), the Kaplan-Meier method and log-rank test were used for statistical analysis as appropriate.

RESULTS: Six months after RIT, hypothyroidism was achieved in 45 (81.8\%), euthyroid state - in 2 (3.6\%), and in 8 (14.6\%) patients thyrotoxicosis persisted. On univariate statistical analysis, the smaller thyroid volume, higher fT4 and lower TSH

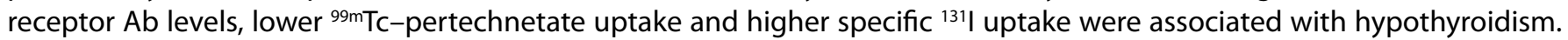
On multivariate logistic regression analysis, the older patient's age $(p=0.011)$, smaller thyroid volume $(p=0.003)$ and higher fT4 $(p=0.024)$ were independent predictors of RIT efficacy. Thyroid volume was also the only variable associated with achievement of hypothyroidism in time after RIT $(p=0.011)$.

CONCLUSION: The efficacy of dosimetry-based RIT in children and adolescents with GD 6 months after treatment was $81.2 \%$. Older patients' age, smaller thyroid volume and higher fT4 level were independent predictors of therapy success. Smaller thyroid volume was also a predictor of the favorable time-related outcome. Statistical models obtained in this work may be used to prospectively estimate the chance of efficient RIT for GD in pediatric patients.

KEYWORDS: radioiodine therapy; children; adolescents; Graves' disease; dosimetry planning; nuclear medicine; theranostics.

\section{ВВЕДЕНИЕ}

Диффузный токсический зоб (ДТЗ), или болезнь Грейвса (БГ), является самой частой причиной гипертиреоза у людей разных возрастных групп, в том числе у детей и подростков, составляя 10-15\% всех заболеваний щитовидной железы (ЩЖ) у детей [1]. Заболеваемость БГ в детской и подростковой возрастной группе 1-2 случая на 100 тысяч человек. Казуистически редко БГ возникает у детей до 5 лет, чаще всего заболевают дети и подростки в возрасте от 11 до 17 лет [2, 3]. К методам лечения БГ у детей и подростков относятся: длительный прием тиреостатиков, хирургическая операция и радиойодтерапия (РЙТ).

При лечении тиреостатическими препаратами (метимазол, пропилтиоурацил) стойкого эффекта удается добиться в 15-40\% случаев, в большинстве случаев в течение 2 лет возникает рецидив тиреотоксикоза [4]. При данном лечении нередко возникают нежелательные явления, как незначительные (зуд, тошнота, артралгии, миалгии и др.), так и опасные для жизни (агранулоцитоз, гепатотоксичность и др.). Риск развития осложнений при приеме тиреостатиков является дозозависимым, токсичность в случае приема метимазола, карбимазола (в РФ не зарегистрирован) и пропилтиоурацила чаще всего перекрестная [5-7].

Оперативное вмешательство в объеме тиреоидэктомии, помимо пожизненной заместительной гормональной терапии препаратами левотироксина натрия и косметического дефекта, сопряжено с достаточно высоким риском повреждения гортанных нервов, гипопаратиреоза, что зависит от квалификации и опыта хирурга [8]. Риск постхирургических осложнений у пациентов с БГ повышен в связи с высокой кровоточивостью ЩЖ ввиду наличия воспаления и зачастую ее спаянностью с окружающими тканями. Самым частым осложнением является транзиторная гипокальциемия - 10-20\%; вероятность развития стойкого гипопаратиреоза - 2\%; образование келоидного рубца - 2,8\%, паралич возвратного гортанного нерва - 2\% [9].
У детей и подростков, как и у взрослых пациентов, первой линией лечения БГ является тиреостатическая терапия на длительный период (как правило, не менее 3 лет) по схеме «блокируй» и/или «блокируй и замещай». При высокой токсичности, неэффективности терапии или низкой дисциплине пациента рассматривается операция или терапия радиоактивным йодом [10].

РЙТ для лечения тиреотоксикоза применяется с 1940-х гг., а у детей и подростков - с середины 1950-х гг. [11]. Опасения эндокринологов при назначении РЙТ чаще всего связаны с ожиданием риска развития радиационно-индуцированных опухолей, однако не опубликовано ни одного доказательного исследования, которое бы это подтверждало [9]. РЙТ для лечения БГ успешно применяется в мире уже более 70 лет, и ее безопасность в различных возрастных группах, в том числе и в детской, подтверждена многочисленными ретроспективными когортными исследованиями [12]. В одном из исследований при анализе отдаленных результатов лечения больных БГ было обнаружено, что риск развития рака ЩЖ в 5 раз выше при лечении тиреостатиками, чем при РЙТ [13].

В клинических рекомендациях Американской тиреоидологической ассоциации и Американской ассоциации клинической эндокринологии рекомендованная лечебная активность ${ }^{131}$ I составляет не менее 150 мкКи на 1 г ткани ЩЖ, а у пациентов с исходным большим объемом ЩЖ (>50 мл) хирургическое лечение предпочтительнее РЙТ [14].

Европейская ассоциация ядерной медицины не рекомендует РЙТ для лечения БГ у детей до 5 лет. У детей, как и у взрослых, активность ${ }^{131}$ I рекомендуется рассчитывать исходя из объема ЩЖ и индивидуальной фармакокинетики йода. При большом объеме ЩЖ (>60 мл) отмечается снижение частоты стойкой ремиссии и предпочтительнее хирургическое лечение [15].

Клинический опыт применения РЙТ в лечении БГ у детей и подростков в мире небольшой, поэтому анализ накопленных в ЭНЦ данных является актуальным. Целью данного исследования является изучение факторов, влияющих на эффективность РЙТ у детей и подростков с БГ. 


\section{ЦЕЛЬ ИССЛЕДОВАНИЯ}

Целью данного исследования является выявление предикторов эффективности лечения радиоактивным йодом среди пациентов младше 18 лет для совершенствования персонализированной тактики лечения.

\section{МАТЕРИАЛЫ И МЕТОДЫ}

В изучаемую группу включены все дети и подростки (в возрасте 8-18 лет) с подтвержденным диагнозом БГ, получившие РЙТ в период с 2016 г. по 2017 г. и имевшие период наблюдения как минимум 6 месяцев после лечения.

Первоначальная выборка включала в себя 61 пациента не старше 18 лет с диагностированной БГ, прошедших РЙТ. Из выборки были исключены 6 случаев с повторным сеансом РЙТ. Таким образом, в исследование были включены 55 пациентов детского и подросткового возраста, получавших РЙТ по поводу БГ первично (рис. 1).

Показания к РЙТ: у 52 пациентов, вошедших в исследование, проводившаяся предварительно медикаментозная терапия оказалась неэффективной; у 3 пациентов наблюдалась непереносимость тиреостатиков (аллергические реакции как на препараты метимазола, так и на препараты пропилтиоурацила); также во всех случаях при выборе между терапией радиоактивным йодом и хирургическим вмешательством (помимо учета исходных данных лабораторного и инструментального исследований) имело место личное предпочтение пациента (или родителей пациента) в пользу неинвазивного метода лечения.

Лиц женского пола - 48, мужского - 7, процентное соотношение Ж:М - 87,3:12,7. Возраст пациентов был от 8 до 18 лет, медиана [квартили] 15 [12; 17] лет.

Анализируемые параметры включали: пол, возраст, объем ЩЖ по данным УзИ до и через 6 месяцев после лечения, статус эндокринной офтальмопатии, длитель-

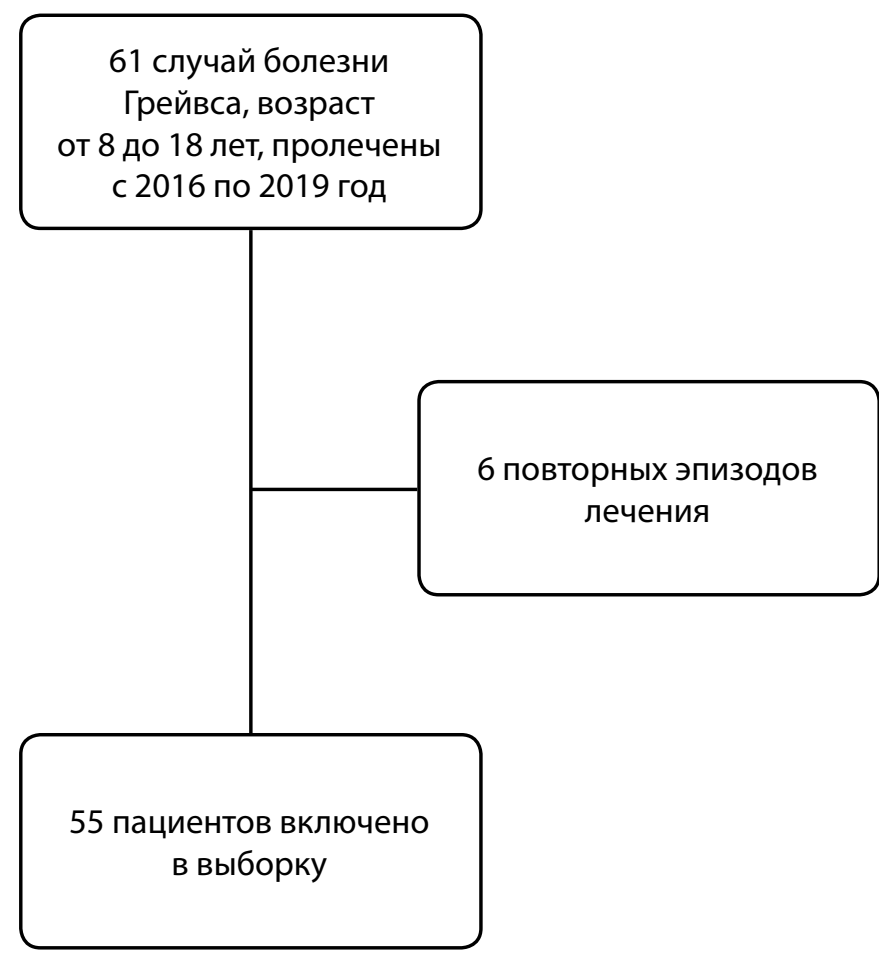

Рис. 1. Процедура отбора случаев болезни Грейвса перед включением в выборку. ность приема тиреостатиков, рецидив тиреотоксикоза на тиреостатической терапии в анамнезе, уровень тиреотропного гормона (ТТГ), свободного Т3 (свТ3), свободного Т4 (свТ4) исходно и на 1, 3, 6-й месяцы после лечения, антитела к рецептору ТТГ исходно и на 3 и 6 месяцы по-

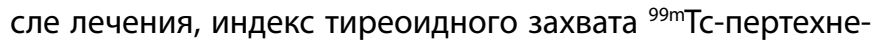
тата на 10-20-й минуте (\%), максимальный тиреоидный захват (\%), время максимального тиреоидного захвата ${ }^{131}$ I (ч), максимальная мощность поглощенной дозы в тиреоидной ткани (Гр/ч), суммарная поглощенная (очаговая) доза (СОД) в течение первых 48 ч после назначения терапевтической активности (Гр).

Всеми пациентами было подписано добровольное информированное согласие на обезличенную обработку данных и их публикацию.

Йод-131 - стерильный раствор натрий йода-131 (производства Института изотопов, Будапешт, Венгрия).

Гамма-камера - система однофотонной эмиссионной компьютерной томографии GE Discovery NM 630. Протокол планарной сцинтиграфии всего тела: скорость стола 30 см/мин; матрица изображения 256×1024; окно

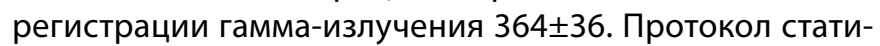
ческой сцинтиграфии: матрица изображения $256 \times 256$; окно регистрации гамма-излучения $364 \pm 36$.

Доз-калибратор - радиометр VDC «Доз-калибратор», ионизационная камера VIK-202. Погрешность измерения $3 \%$.

узИ-аппараты. Toshiba Aplio 500, линейный датчик PLT-I204BX с диапазоном частот 7-18 МГц, Aloka prosound alpha 10, линейный датчик UST-5543 с диапазоном частот 4-13 МГц; Аccuvix A 30, линейный датчик L5-13IS с диапазоном частот 5-13 МГц; Medison SonoAce R5, линейный датчик LN5-12 с диапазоном частот 5,0-12,0 МГц; Esaote MyLab 20, линейный датчик LA523 с диапазоном частот 4-13 МГц. Исследование выполнялось в стандартном положении пациента лежа на спине с запрокинутой головой и подложенным под плечи валиком. Осуществлялось сканирование ЩЖ в В-режиме и с применением режима цветового допплеровского картирования (ЦДК). Производилось измерение трех размеров обеих долей щЖ (длина/ширина/переднезадний размер) для вычисления объема щЖ по формуле J. Brunn (1981 г.). Оценивалась структура ЩЖ, степень снижения эхогенности и усиления васкуляризации, наличие узловых образований.

(TU) Индекс тиреоидного захвата 99mТс-пертехнетата [\%] - отношение накопленной активности 99mТс-пертехнетата в ЩЖ $\mathrm{A}_{\text {щж }}^{99 m T c}$ [МБк] на 10-20-й минуте (с учетом распада на момент $t_{1}$ [мин] исследования) к активности 99mТс-пертехнетата $\mathrm{A}_{0}^{99 m T c}$ [МБк], введенной пациенту в момент времени $t_{0}[$ мин] внутривенной инъекции ${ }^{99 \mathrm{~m}}$ Тс-пертехнетата:

$$
\boldsymbol{T} \boldsymbol{U}=\frac{\mathrm{A}_{\amalg}^{99 m T c} \cdot e^{\frac{\left(t_{1}-t_{0}\right)}{6 \cdot 01}} \cdot \ln 2}{A_{0}^{99 m T c}} .
$$

(IU $)_{\max }$ ) Максимальный тиреоидный захват 131। [\%] - отношение активности ${ }^{131}$ в в ЩЖ на пике его накопления $\max \left(A_{щ ж}^{\text {тр }}\right)$ [МБк] к введенной трейсерной активности $A_{0}^{\text {Tp }}\left[\mathrm{MБK}{ }^{131}\right.$ :

$$
\boldsymbol{I} \boldsymbol{U} \max =\frac{\max \left(\mathrm{A}_{\text {щж }}^{\mathrm{Tp}}\right)}{\mathrm{A}_{0}^{\mathrm{Tp}}} .
$$




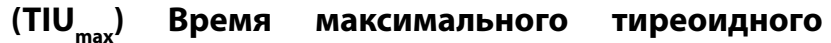
захвата ${ }^{131}$ I [4] - время пика накопления активности ${ }^{131}$ ] в ЩЖ от момента введения трейсерной активности $\mathrm{A}_{\text {щж }}^{\mathrm{Tp}}$ [MБK]:

$$
\boldsymbol{T I} \boldsymbol{U}_{\boldsymbol{m a x}}=T\left(\max \left(\mathrm{A}_{щ ж}^{\mathrm{Tp}}\right)\right) .
$$

$\left(D R_{\max }\right)$ Максимальная мощность поглощенной дозы в тиреоидной ткани [Гр/ч] - максимальная поглощенная энергия за час в ткани щЖ после введения терапевтической активности $A_{0}^{\text {тер }}$ [ГБК], отнесенная к объему ЩЖ $V_{\text {щж: }}$

$$
\boldsymbol{D} \boldsymbol{R}_{\max }=\frac{A_{0}^{\mathrm{Tep}} \cdot I U \max \cdot 0,108}{V_{\text {Шж}}} .
$$

$\left(\operatorname{RAD}_{48 \mathrm{~h}}\right)$ Суммарная очаговая поглощенная доза в течение первых 48 ч [Гр] - суммарная поглощенная энергия в ткани ЩЖ в течение 48 ч после введения терапевтической активности ${ }^{131} A_{0}^{\text {тер }}$ [ГБК], отнесенного к объему ЩЖ $V_{\text {щж: }}$

$$
\boldsymbol{R} \boldsymbol{A} \boldsymbol{D}_{\mathbf{4 8 \boldsymbol { h }}}=\frac{A_{0}^{\mathrm{Tep}}}{A_{0}^{\mathrm{Tp}}} \frac{\int_{0}^{48} A(t)_{\text {щж }}^{\mathrm{Tp}} d t \cdot 0,108}{V_{\text {шж }}} .
$$

Среда моделирования источника ${ }^{131} \mathrm{I}$ - GATE 7.2. Число разыгрываемых ионов: 1 млн. Геометрия модели: эллипсоид. Распределение источника: изотропное. Физика: EmLivermore.

Расчет индивидуальной терапевтической активности ${ }^{131}$ в выполнялся с учетом индивидуальной кинетики трейсерной активности (5-10 МБк перорально) ${ }^{131}$ I. Сцинтиграфия выполнялась на 1-2, 5-6, 23-25, 47-49 ч. Первое и последнее исследование проводились в режиме «все тело», 2-е и 3-е - в режиме статической сцинтиграфии щЖ. Результаты подвергались обработке в режиме заданной отсечки 65\%, 86\%, 86\%, $86 \%$ соответственно. Производился пересчет счета с гамма-камеры в активность с помощью коэффициента, полученного в результате отнесения введенной трейсерной активности к счету от всего тела на первой планарной сцинтиграфии с учетом фонового счета, получаемого в отсутствие источника. По полученным данным проводилась аппроксимация теоретической биэкспоненциальной зависимостью с расчетом коэффициентов. По полученной зависимости рассчитывался вектор А активности ${ }^{131}$ в щ Щ с указанием значения на каждый час после введения. Вектор активности использовался для определения индивидуальной активности ${ }^{131}$ I при РЙТ тиреотоксикоза, данные оцениваются за первые 48 ч измерений. Для этого в векторе определяется максимальное значение активности ${ }^{131} \mathrm{I}, A^{\max }=\max (\mathrm{A})$ [ГБк - ч]. Далее определяют максимальный тиреоидный захват ${ }^{131}$, с учетом введенной пероральной активности $\mathrm{A}_{0}$, по формуле:

$$
\mathrm{MU}=\frac{A^{\max }}{A_{0}} .
$$

Интегральный захват рассчитывался по формуле:

$$
\mathrm{IU}=\frac{\sum_{1}^{48} \mathrm{~A}(t)}{\sum_{1}^{48} A_{0}(t)},
$$

интеграл тиреоидного захвата ${ }^{131}$ I за период от введения до 48 часов после введения. Лечебную активность тиреоидной ткани $A_{\text {тт }}^{k}$ при удельном индексе тиреоидного захвата $99 \mathrm{~m}$ Тс-пертехнетата менее 0,5 \%/мл определяют по формуле:

$$
A_{\mathrm{TT}}^{k}=\min \left(\frac{1,5 \cdot V_{k}}{M U \cdot R_{k} \cdot S_{0}}, \frac{55 \cdot V_{k}}{I U \cdot R_{k} \cdot S_{0}}\right),
$$

более 0,5 \%/мл - по формуле:

$$
A_{\mathrm{TT}}^{k}=\min \left(\frac{2,0 \cdot V_{k}}{M U \cdot R_{k} \cdot S_{0}}, \frac{73 \cdot V_{k}}{I U \cdot R_{k} \cdot S_{0}}\right),
$$

где $S_{0}=0,108\left[\frac{\text { Гр'мл }}{\text { ГБкчч }}-\right.$ фактор накопления дозы. Индивидуальная терапевтическая активность ${ }^{131}$ I определялась по формуле:

$$
\mathrm{A}_{\text {инд }}=\max \left(A_{\text {тТ }}^{k}\right) \text {. }
$$

Алгоритм расчета индивидуальной активности терапевтической активности ${ }^{131}$ І для РЙТ БГ у детей и подростков представлен на рисунке 2.

\section{Статистическая обработка}

Описательная статистика количественных признаков представлена медианой и квартилями в виде Mediana [Qн; Qв]. В одномерных статистических расчетах использовали точный тест Фишера для бинарных переменных и непараметрический тест Манна-Уитни для непрерывных независимых выборок или тест знаковых рангов Уилкоксона для парных выборок.

В многомерном анализе результатов РЙТ использовали логистическую регрессионную модель. В модель включали состояние гипотиреоза через 6 месяцев после РЙТ в качестве зависимой переменной, а в качестве объясняющих переменных - параметры, известные до начала лечения: пол, возраст, объем ЩЖ, наличие рецидива на отмену тиреостатической терапии, исходный уровень свТ4. Модель строилась методом пошагового отбора переменных. Выполнялись перекрестная проверка полученной модели способом скользящего контроля по отдельным объектам и ROC-анализ модели и независимых предикторов, входящих в нее. Отрезную точку определяли по критерию Юдена [16].

Для изучения достижения состояния гипотиреоза после РЙТ во времени строили модель пропорциональных рисков (регрессия Кокса), используя метод пошагового отбора переменных. На вход пошаговой процедуры подавали как параметры, известные до начала лечения: пол, возраст, объем щЖ, наличие рецидива на отмену тиреостатической терапии, исходный уровень свТ4, так и параметры, определенные в курсе РЙт: индекс тиреоидного захвата ${ }^{99 m}$ Тс-пертехнетата и удельный захват ${ }^{131} \mathrm{I}$. Оценку модели времени до события проводили методом Каплана-Майера. Сравнение распределений времени до события между подгруппами проводили с помощью логарифмического рангового теста.

Для расчетов использовали 3.71 release of SAS Studio for the 9.4M5 version of SAS, JMP Pro 15.1.0. и SPSS v.24. Уровнем статистической значимости традиционно считали $\mathrm{p}<0,05$.

\section{РЕЗУЛЬТАТЫ}

Через 6 месяцев после лечения 55 пациентов гипотиреоз развился у 45 (81,8\%), эутиреоз - у 2 (3,6\%) и у $8(14,6 \%)$ тиреотоксикоз сохранился. Сравнительные характеристики подгрупп пациентов с достигнутым через 6 месяцев после РЙт гипотиреозом и таковых в состоянии эутиреоза или гипертиреоза приведены в таблице 1. 


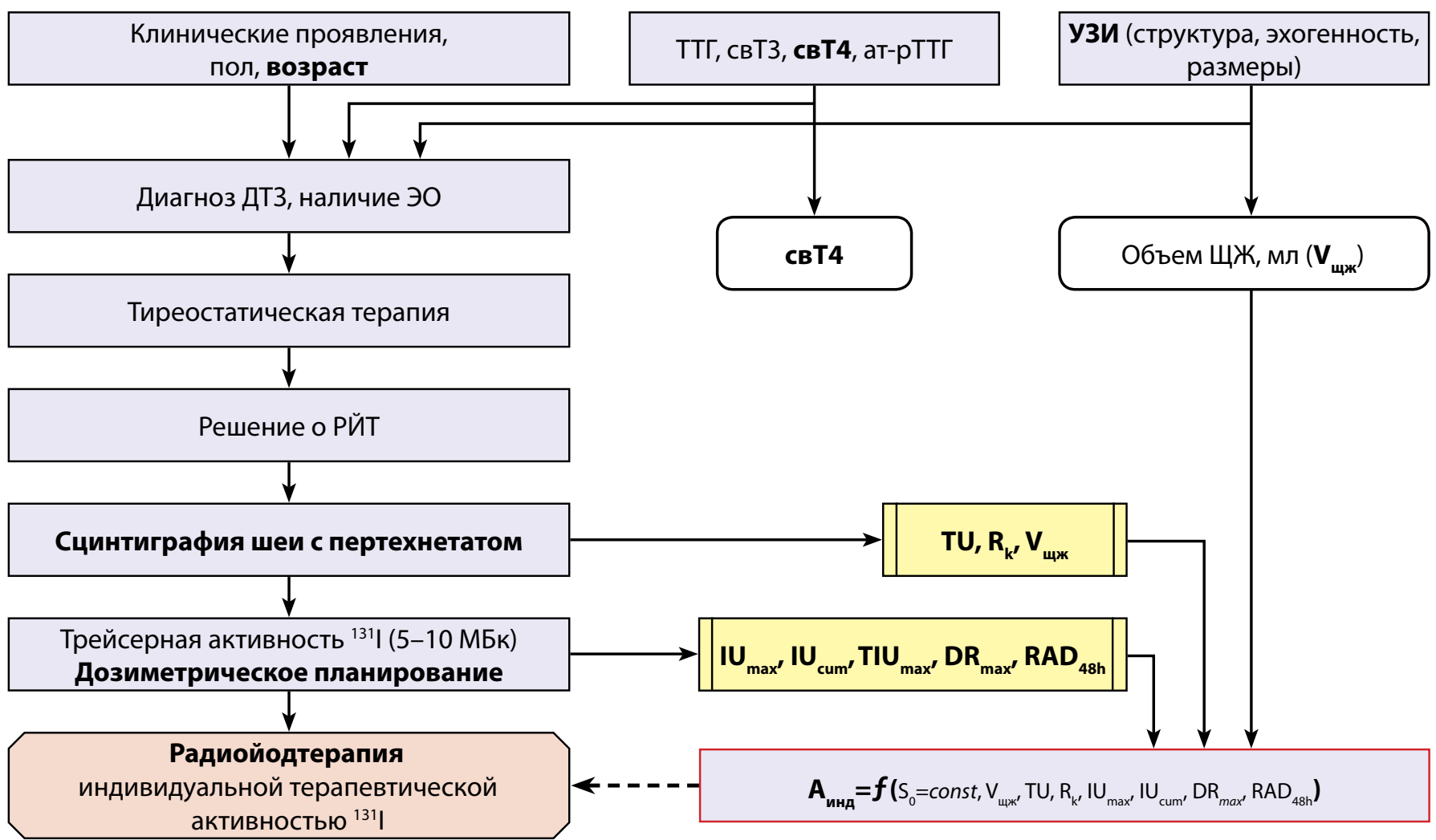

Рис. 2. Алгоритм расчета индивидуальной активности терапевтической активности ${ }^{131}$ для радиойодтерапии болезни Грейвса у детей и подростков.

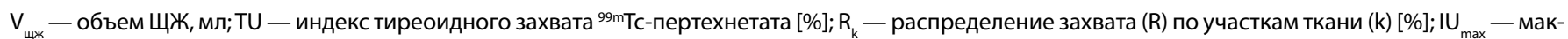
симальный тиреоидный захват ${ }^{131}$ [ [\%]; IU сum - кумулятивный тиреоидный захват ${ }^{131}$ [ [\%]; $\mathrm{TIU}_{\max }$ - время максимального тиреоидного захвата ${ }^{131}$ [ч]; $\mathrm{DR}_{\text {max }}$ - максимальная мощность поглощенной дозы в тиреоидной ткани [Гр/ч]; $\mathrm{RAD}_{48 \mathrm{~h}}$ - суммарная очаговая поглощенная доза в течение первых 48 ч [Гр]; $\mathrm{A}_{\text {инд }}$ - индивидуальная терапевтическая активность ${ }^{131} 1 ; \mathrm{S}_{0}$ - фактор накопления дозы; ЭО — эндокринная офтальмопатия.

Таблица 1. Сравнительные исходные характеристики группы пациентов с достигнутым через 6 месяцев после радиойодтерапии гипотиреоидным статусом и группы, не ответившей на лечение.

\begin{tabular}{|c|c|c|c|c|}
\hline Предикторы & Bce & Гипотиреоз & $\begin{array}{c}\text { Гипертиреоз/ } \\
\text { Эутиреоз }\end{array}$ & $\mathbf{p}$ \\
\hline Всего & $55(100 \%)$ & $45(82 \%)$ & $10(18 \%)$ & \\
\hline \multicolumn{5}{|l|}{ Пол } \\
\hline Муж. & $7(13 \%)$ & $7(14 \%)$ & $0(0 \%)$ & \multirow{2}{*}{0,328} \\
\hline Жен. & $48(87 \%)$ & $38(86 \%)$ & $10(100 \%)$ & \\
\hline Возраст, годы ${ }^{1}$ & $15,0[12,0 ; 17,0]$ & $15,0[11,5 ; 17,0]$ & $14,5[12,8 ; 17,0]$ & 0,895 \\
\hline Объем ЩЖ, мл ${ }^{1}$ & $29,0[21,2 ; 47,0]$ & $26,6[18,6 ; 36,0]$ & $50,9[46,5 ; 87,5]$ & 0,001 \\
\hline Офтальмопатия & $14(32 \%), n=44^{2}$ & $9(26 \%), n=34$ & $5(50 \%)$ & 0,247 \\
\hline $\begin{array}{l}\text { Длительность тиреостатической } \\
\text { терапии, мес }^{1}\end{array}$ & $\begin{array}{c}34,0[19,0 ; 54,0] \\
n=51\end{array}$ & $\begin{array}{c}34,5[22,3 ; 55,3] \\
n=42\end{array}$ & $\begin{array}{c}21,0[15,5 ; 57,0] \\
n=9\end{array}$ & 0,387 \\
\hline $\begin{array}{l}\text { Рецидив тиреотоксикоза } \\
\text { при снижении дозы тиреостатика }\end{array}$ & $22(40 \%)$ & $15(33 \%)$ & $7(70 \%)$ & 0,070 \\
\hline Свободный Т4, пM/л'1 & $12,7[9,1 ; 16,9]$ & $13,7[10,0 ; 17,1]$ & $9,8[5,1 ; 12,6]$ & 0,016 \\
\hline Свободный Т3, пМ/л' & $5,7[4,2 ; 7,8], n=54$ & $5,5[4,3 ; 7,8], n=44$ & $6,2[4,1 ; 9,3]$ & 0,894 \\
\hline ТТГ, мЕд/л ${ }^{1}$ & $\begin{array}{c}0,036[0,008 ; 0,978] \\
n=54\end{array}$ & $\begin{array}{c}0,036[0,010 ; 1,480] \\
n=44\end{array}$ & $0,045[0,005 ; 0,395]$ & 0,367 \\
\hline Антитела к рецептору ТТГ, Ед/л' & $8,8[3,3 ; 20,0], n=47$ & $7,7[3,0 ; 16,3], n=37$ & $25,0[5,3 ; 40,0]$ & 0,037 \\
\hline Индекс захвата 99mТс-пертехнетата, \%1 & $0,14[0,08 ; 0,22]$ & $0,13[0,08 ; 0,19]$ & $0,35[0,11 ; 0,38]$ & 0,042 \\
\hline Назначенная активность ${ }^{131}$, МБк ${ }^{1}$ & $882[744 ; 1084]$ & $871[742 ; 1030]$ & $1060[784 ; 1155]$ & 0,138 \\
\hline Максимальный захват ${ }^{131}$ I, \%1 & $0,45[0,39 ; 0,50]$ & $0,44[0,35 ; 0,50]$ & $0,47[0,44 ; 0,54]$ & 0,084 \\
\hline Удельный захват ${ }^{131}$ I, МБк/г & $13,8[11,3 ; 16,6]$ & $14,6[11,5 ; 17,8]$ & $9,9[6,7 ; 11,9]$ & 0,001 \\
\hline
\end{tabular}

${ }^{1}$ медианное значение и [квартили].

$2 \mathrm{n}$ - число пациентов с информацией по данному параметру; все пациенты включены, если $\mathrm{n}$ не указано. 


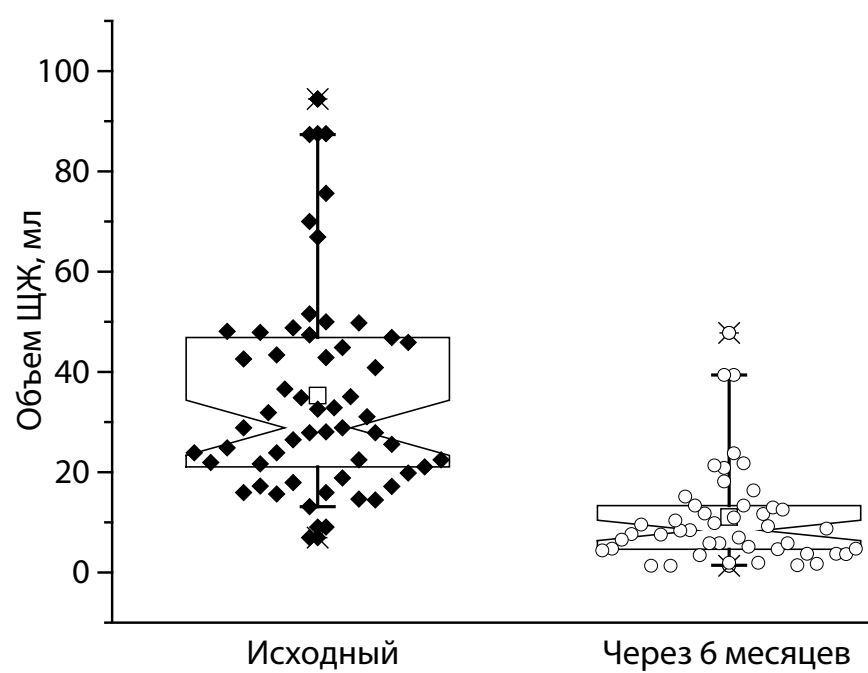

Рис. 3. Диаграмма объема щитовидной железы до и после проведения радиойодтерапии. Верхняя и нижняя граница прямоугольников с вырезами соответствуют 75 и 25 процентилям, вырезы - 50 процентилю (медианному значению), квадрат внутри - среднему значению. Горизонтальные черточки соответствуют 95 процентилю, а 1 и 99 процентили указаны крестиками. Снижение объема щЖ после лечения было статистически значимым, p<0,001 согласно критерию Уилкоксона для связных выборок.

Уменьшение объема ЩЖ спустя 6 месяцев после РЙт варьировало у различных пациентов от $12,2 \%$ до $94,3 \%$ (72,8 [58,8; 82,4]\%); уменьшение объема было статистически значимым ( $<<0,001$, рис. 3). Статистически значимой динамики титра антител к рецептору ТТГ через 6 месяцев не отмечено (исходно: 8,8 [3,6; 29,0] Ед/л; через 6 месяцев: $11,2[4,0 ; 24,4]$ Ед/л; $p=0,564)$.

Результат моделирования достижения гипотиреоза через 6 месяцев после РЙТ с помощью логистического регрессионного анализа показал, что независимыми предикторами исхода являются возраст пациента, а также объем ЩЖ и уровень свТ4 до начала лечения (табл. 2). Полученные оценки эффектов позволяют считать более старший возраст $(p=0,011)$, меньший объем ЩЖ $(p=0,003)$ и более высокий уровень свТ4 $(p=0,024)$ факторами, повышающими вероятность достижения гипотиреоза. По результатам ROC-анализа, полученная оценка площади под кривой (AUC=0,942 (95\% ДИ 0,883-1,000)), а также высокие значения оценок чувствительности (0,822 (95\% ДИ 0,687-0,907)), специфичности $(1,000$ (95\% ДИ 0,722-1,000)) и показателя прогностической ценности положительного результата $(1,000(95 \%$ ДИ 0,906-1,000)) в отрезной точке (оценка $\mathrm{p}=0,857)$ предполагают достаточно высокий потенциал модели в предсказании желательного исхода лечения. Довольно низкая оценка показателя прогностической ценности отрицательного результата (0,556 (95\% ДИ 0,337-0,754)), вероятно, связана с относительно небольшим количеством пациентов, не достигших гипотиреоза в исследованной группе. Перекрестная проверка продемонстрировала лишь небольшую степень переоценки эффективности полученной модели (AUC=0,913 (95\% ДИ 0,836-0,991)).

Поскольку достижение гипотиреоза наблюдалось через 1, 3 и 6 месяцев после РЙТ у разных пациентов, для определения независимых предикторов результата лечения во времени была применена регрессия Кокса. Единственным статистически значимым параметром оказался исходный объем ЩЖ, оценка эффекта которого составила $\mathrm{HR}=0,976$ (95\% ДИ 0,958-0,995; $\mathrm{p}=0,011)$. Согласно этой модели, шанс достижения гипотиреоза на 2,4\% ниже на каждый дополнительный миллилитр объема ЩЖ в любой момент времени с начала лечения (до по крайней мере 6 месяцев). С учетом вариабельности объема щЖ в исследованной группе можно ожидать, что на каждый дополнительный миллилитр объема шанс достижения гипотиреоза может снижаться от 0,5\% до 4,2\%. Базовый кумулятивный риск (или, применительно к данному исследованию, - шанс достижения гипотиреоза) составил 1,042 для 1 месяца, 3,234 для 3 месяцев и 4,369 для 6 месяцев. Эти параметры в совокупности с регрессионным коэффициентом для объема щЖ $(-0,24 \pm 0,010$ ( \pm стандартная ошибка)) могут быть использованы для оценки шанса достижения гипотиреоза пациентом в данные моменты времени после начала лечения.

В качестве иллюстрации связи между объемом ЩЖ ишансом достижения гипотиреоза во времени после начала лечения нами была проанализирована функция выживаемости (т.е. достижение гипотиреоза) у пациентов, стратифицированных по исходному объему щЖ. С помощью ROC-анализа было установлено, что пороговым является объем ЩЖ 43 мл (AUC=0,927 (95\% ДИ 0,859-0,995)). На рисунке 4 представлены доли пациентов, достигших

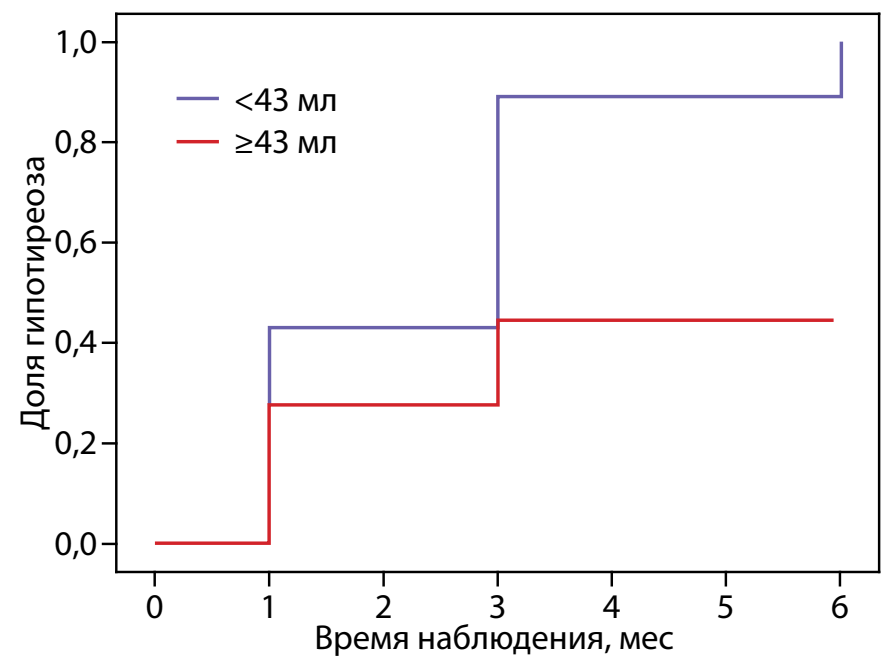

Рис. 4. Кумулятивная доля пациентов с исходным объемом щитовидной железы менее или более 43 мл, достигших гипотиреоза в разные сроки после радиойодтерапии, оцененная методом Каплана-Майера. Различие было статистически значимым ( $<00,001$, логарифмический ранговый тест).

Таблица 2. Многомерная логит-регрессионная модель достижения гипотиреоза через 6 месяцев после радиойодтерапии'.

\begin{tabular}{lcccc}
\hline \multicolumn{1}{c}{ Предикторы } & Коэффициент $\boldsymbol{\beta}_{\mathbf{i}}$ & Ст. ошибка & $\begin{array}{c}\text { Отношение } \\
\text { шансов (OR) }\end{array}$ & 95\% дИ для ОR \\
\hline Возраст, годы & 0,266 & 0,105 & 1,305 & $1,063-1,602$ \\
Объем ЩЖ, мл & $-0,111$ & 0,037 & 0,895 & $0,832-0,963$ \\
СвТ4 перед РЙТ, пМ/л & 0,210 & 0,093 & 1,234 & $1,028-1,481$ \\
\hline
\end{tabular}

${ }^{1}$ Модель не включала константу $\left(\beta_{0}=0\right) ;$ критерий согласия Хосмера-Лемешова $\mathrm{p}=0,593$. 
состояния гипотиреоза в различное время после РЙТ в подгруппах с объемом ЩЖ <43 мл или $\geq 43$ мл; различие между подгруппами было статистически значимым ( $<<0,001$, логарифмический ранговый тест).

Из дополнительных наблюдений за рассматриваемой группой пациентов отметим следующие. В течение 1-й недели после РЙТ у 11 (20\%) пациентов отмечался умеренный болевой синдром в области шеи длительностью 7-10 дней. У 3 (6,8\%) из 44 пациентов с установленной до РЙТ неактивной формой офтальмопатии после лечения отмечалось кратковременное ухудшение в виде прогрессирования экзофтальма с последующим самопроизвольным улучшением и у 1 (5,6\%) пациента - ухудшение, купированное сеансом пульс-терапии преднизолоном. У 1 (5,6\%) ребенка отмечено улучшение глазной симптоматики.

У 4 детей, не ответивших на первую РЙТ, лечение было проведено повторно с развитием гипотиреоза у 3 пациентов и эутиреоза еще в одном случае.

\section{ОБСУЖДЕНИЕ}

\section{Резюме основного результата исследования}

В исследовании изучено влияние на достижение гипотиреоза после проведения РЙТ у детей и подростков с БГ таких факторов, как пол, возраст, объем ЩЖ, наличие или отсутствие офтальмопатии, длительность тиреостатического лечения, наличие рецидива на фоне приема тиреостатика, уровень ТТГ, свТ3, свТ4 исходно и на 1, 3, 6-й месяц, АТ-ТТГ исходно и на 3 и 6-й месяц, захват ЩЖ 99mТс-пертехнетата на 20-й минуте, максимальное накопление ${ }^{131} \mathrm{I}$, время максимального накопления ${ }^{131}$ I, максимальная мощность поглощенной дозы, поглощенная доза в течение первых 48 ч после введения терапевтической активности ${ }^{131} \mathrm{I}$. Статистически значимыми в логит-регрессионной модели прогностическими факторами являются: объем ЩЖ (OR=0,895; 95\% ДИ 0,832-0,963; $p=0,003)$, возраст пациента $(\mathrm{OR}=1,305 ; 95 \%$ ДИ 1,063-1,602; $\mathrm{p}=0,011)$ и уровень свТ4

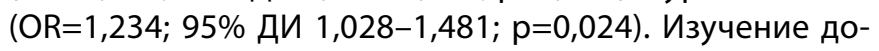
стижения гипотиреоза во времени показало, что единственным статистически значимым параметром оказался исходный объем ЩЖ, HR=0,976 (95\% ДИ 0,958-0,995; $\mathrm{p}=0,011)$. Представленные параметры статистических моделей позволяют оценивать вероятность достижения благоприятного исхода лечения.

\section{Обсуждение основного результата исследования}

Подтверждение нами исходного объема ЩЖ в качестве важнейшего предиктора РЙТ БГ согласуется с выводами Американской тиреоидологической ассоциации и Американской ассоциации клинических эндокринологов, которая выделяет большой объем ЩЖ (50-80 мл) в качестве фактора недостаточной эффективности терапии радиоактивным йодом, что может потребовать повторного проведения РЙТ, и рекомендует рассматривать в подобных случаях альтернативу в виде хирургического лечения [14]. Аналогичных взглядов придерживается Европейская ассоциация ядерной медицины, диктуя необходимость расчета активности ${ }^{131}$, исходя из индивидуальных характеристик пациента с учетом объема щЖ [15].
Согласно большинству опубликованных источников литературы, наибольшее значение придается таким параметрам, как исходный объем ЩЖ, терапевтическая удельная активность ${ }^{131}$ и и суммарная очаговая поглощенная доза.

В 2003 г. Rivkees и соавт. опубликовали результаты исследования, согласно которым частота гипотиреоза составила 50\%, 70\% и 95\%, при лечении активностью ${ }^{131}$ I из расчета на СОД в щЖ 100 Гр ( 110 мкКи/г), 200 Гр ( 220 мкКи/г) и 300 Гр ( 330 мкКи/г ЩЖ) соответственно. Для достижения стойкого гипотиреоза авторы рекомендуют выполнять лечение удельной активностью ${ }^{131}$ | из расчета 300 мкКи/г [17], что не противоречит рекомендациям ATA: не менее 150 мкКи ${ }^{131}$ I на 1 г ткани ЩЖ (около 95\% достижения гипотиреоза) [14]. Kaplowitz и соавт. опубликовали в 2019 г. результаты лечения радиоактивным йодом 78 детей и подростков (от 6 до 19 лет) с БГ [18]. Гипотиреоз был достигнут в 73\% случаев. Эффективность РЙТ изучалась не только в течение 6 мес, но и в более поздние сроки - год и более. Необходимо отметить, что у части пациентов эффект был достигнут за пределами полугода наблюдения после РЙТ. Меньшая масса щЖ (36,5 против 47,4 г; $p=0,037)$ и более высокая удельная терапевтическая активность ${ }^{131}$ I (242 против 212 мкКи/г ткани ЩЖ; $p=0,013$ ) статистически значимо влияли на эффективность лечения, что в целом совпадает с выводами настоящей работы.

В нашем исследовании как объем (масса) ЩЖ, так и удельная активность ${ }^{131}$ І статистически значимо влияли на эффективность лечения. Однако последний фактор непосредственно связан с объемом ЩЖ, влияние которого и оказывается наиболее значимым при выборе РЙТ в качестве тактики лечения. Несмотря на то что удельная активность ${ }^{131}$ І является статистически значимым параметром ( $p<0,001$ в одномерном анализе), в данном исследовании мы не верифицировали его в качестве независимого предиктора в логитрегрессионной модели.

Параметр «возраст» многомерной логистической регрессионной модели был подтвержден как статистически значимый. Ранее данный параметр уже изучался. Так, в исследовании из Таиланда (2013 г.), в котором 27 детям и подросткам (средний возраст 14,6 лет) была проведена РЙТ активностью ${ }^{131}$ из расчета 150 мкКи на 1 г тиреоидной ткани, через 6 месяцев зарегистрирована частота посттерапевтического гипо- и эутиреоза 55,6\% [19]. В исследовании сравнивалось влияние возраста, пола, 2-х и 24-часовой захват ${ }^{131} \mathrm{I}$, объем ЩЖ и суммарная доза облучения ЩЖ, но значимых различий между группами пациентов, достигших и не достигших гипотиреоидного статуса, выявлено не было. Однако необходимо принять во внимание малочисленность клинической выборки в упомянутом исследовании. На наш взгляд, рациональным подходом будет перепроверка прогностической значимости возраста на более многочисленной выборке, так как на сегодняшний день фактор возраста детей и подростков изучен недостаточно, особенно в контексте пубертата.

Также при анализе влияния факторов в прогностической модели в нашей выборке было обнаружено, что важным предиктором эффективности РЙТ является уровень свТ4. В исследовании от 2009 г., включавшем 
38 пациентов в возрасте от 30 дней до 21 года, отмечалась связь уровня свТ4 с ответом на РЙТ [20]. Помимо этого, авторы исследования выделили в качестве предикторов неэффективности РЙТ наличие в анамнезе применения тиреостатиков (37\% против 0\%; $p=0,02)$, офтальмопатии (58\% против 8\%; $p=0,002)$ и интервал более 12 месяцев от постановки диагноза до РЙТ (50\% против $10 \% ; p=0,003)$. В нашем исследовании связи тиреостатической терапии и наличия офтальмопатии с результатом РЙТ выявлено не было (см. табл. 1), а интервал между постановкой диагноза БГ и началом РЙТ не исследовался. Наши результаты, полученные на большем количестве пациентов, возраст которых, вероятно, несколько отличался от такового в рассматриваемой работе, не дают достаточных оснований считать применение тиреостатиков и наличие офтальмопатии независимыми предикторами эффективности РЙТ.

По нашему мнению, учет объема ЩЖ и уровня свТ4 имеет важнейшее практическое значение, поскольку эти показатели влияют на решение о назначении тиреостатиков. На фоне многолетней тиреостатической терапии объем щЖ чаще всего увеличивается, тем самым снижая потенциал эффективности РЙТ, что свидетельствует в пользу рациональности сокращения длительности терапии тиреостатиками. С этой точки зрения следует рассматривать РЙТ как наиболее оптимальный вариант лечения с высокой эффективностью при благоприятном индивидуальном сочетании прогностических факторов.

В целом представленные в нашей работе результаты, а именно параметры регрессионных моделей, могут быть использованы на практике для проспективной оценки вероятности эффективности РЙТ у пациентов детско-подросткового возраста, страдающих БГ. В случае получаемой высокой оценки вероятности рекомендация к проведению РЙТ получает дополнительные основания, в то время как низкая оценка может служить рекомендацией к изменению тактики (например, повышение дозы ${ }^{131}$ I) или даже стратегии лечения (например, в пользу хирургического подхода). Знание ключевыххарактеристик, значимо влияющих на успех РЙТ, может также служить отправной точкой для создания более сложных предсказательных алгоритмов, например, с использованием технологий машинного обучения.

\section{Ограничения исследования}

Ограничениями нашего исследования являются недостаточно многочисленная выборка и отсутствие оценки комплаентности пациентов в части приема тиреостатической терапии. Период наблюдения в пределах 6 месяцев также являлся ограничением, так как часть эффектов могла реализовываться в более поздние сроки.

\section{ЗАКЛЮЧЕНИЕ}

Эффективность РЙТ с использованием дозиметрического подхода при ее планировании у детей и подростков с БГ в нашем исследовании составила $81,8 \%$ согласно результатам наблюдения пациентов на протяжении 6 месяцев после лечения. Основными прогностическими факторами эффективности лечения являются объем ЩЖ c OR=0,895 (95\% ДИ 0,832-0,963; $p=0,003)$, возраст пациента с OR=1,305 (95\% ДИ 1,063-1,602; $p=0,011)$ и уровень свT4 с OR=1,234 (95\% ДИ 1,028-1,481; $=0,024)$. Анализ эффекта РЙТ во времени позволил установить меньший объем ЩЖ как фактор более благоприятного исхода лечения $\mathrm{HR}=0,976$ (95\% ДИ 0,958-0,995, $\mathrm{p}=0,011)$. Представлены параметры логистической регрессионной модели и модели пропорциональных рисков, на основании которых возможно соответственно оценивать вероятность достижения благоприятного исхода лечения в течение полугода или на 1, 3 и 6 месяцев. Необходимо продолжить совершенствование алгоритмов индивидуального дозиметрического планирования и изучение предикторов эффективности терапии радиоактивным йодом в различных возрастных группах на более многочисленной и длительно прослеженной выборке пациентов.

\section{ДОПОЛНИТЕЛЬНАЯ ИНФОРМАЦИЯ}

Источник финансирования. Поисково-аналитическая работа и подготовка статьи проведены на личные средства авторского коллектива.

Конфликт интересов. Все авторы декларируют отсутствие явных и потенциальных конфликтов интересов, связанных с публикацией настоящей статьи.

Участие авторов. Все авторы принимали участие в наблюдении пациента, внесли значимый вклад в подготовку статьи, прочли и одобрили финальную версию перед публикацией.

\section{СПИСОК ЛИТЕРАТУРЫ | REFERENCES}

1. Kraiem Z, Newfield RS. Graves' disease in childhood. J Pediatr Endocrinol Metab. 2001;14(3):229-243. doi: 10.1515/jpem.2001.14.3.229.

2. Muratova ST, Ismailov SI. Mental characteristics of teenagers with Graves' disease in Uzbekistan. European science review (Vienna). 2015;7-8:77-80.

3. Чернышова В.С., Стольникова Т.Г., Евтухова О.В., Кулакова Е.Н. Особенности клинической картины диффузного токсического зоба у детей Воронежской области // Медицина: теория и практика. - 2019. - T.4. - №S. - C. 601. [Chernyshova VS, Stol'nikova TG, Evtukhova OV, Kulakova EN. Osobennosti klinicheskoy kartiny diffuznogo toksicheskogo zoba u detey Voronezhskoy oblasti. Meditsina: teoriya i praktika. 2019;4(S):601. (In Russ.)].

4. Glaser NS, Styne DM. Organization of Pediatric Endocrinologists of Northern California Collaborative Graves' Disease Study G. Predicting the likelihood of remission in children with Graves' disease: a prospective, multicenter study. Pediatrics. 2008;121(3):481-488. doi: 10.1542/peds.2007-1535
5. Takaya K, Kimura N, Hiyoshi T. Antithyroid arthritis syndrome: a case report and review of the literature. Intern Med. 2016:55(24):3627-3633. doi: 10.2169/internalmedicine.55.7379.

6. Nikitopoulou E, Gazi S. Antithyroid drug induced syndrome that lies in between ANCA vasculitis and lupus-like syndrome in a 40-year-old female with Graves' disease under methimazole therapy: A Case report. Mediterr J Rheumatol. 2018:19;29(1):52-54. doi: 10.31138/mjr.29.1.52.

7. Ismailov SI, Khayitboeva KK. Problems of diagnostics and treatment of diffuse - toxic goiter (Graves' disease). Vestnik of Saint Petersburg University. Medicine. 2019;14(2):98-104. doi: 10.21638/spbu11.2019.202.

8. Румянцев П.О., Шеремета М.С., Кияев А.В., и др. Эффективность и безопасность радиойодтерапии диффузного токсического зоба (болезни Грейвса) у детей и подростков // Клиническая и экспериментальная тиреоидология. — 2017. — T.13. — №1. C. 6-11. [Rumiantsev PO, Sheremeta MS, Kiyaev AV, et al. Efficacy and safety of radioiodine treatment of Graves' disease in children and adolescents. Clinical and experimental thyroidology. 2017;13(1):6-11. (In Russ.)]. doi: 10.14341/ket201716-11. 
9. Безлепкина О.Б. Федеральные клинические рекомендации по диагностике и лечению болезни Грейвса у детей // Проблемы эндокринологии. - 2014. - Т.60. - №3. - С. 59-68. [Bezlepkina OB. Federal clinical guidelines for the diagnosis and treatment of Graves' disease in children. Problems of Endocrinology. 2014;60(3):59-68 (In Russ.)]. doi: 10.14341/probl201460359-68.

10. Kahaly GJ, Bartalena L, Hegedüs L, et al. 2018 European thyroid association guideline for the management of graves' hyperthyroidism Eur Thyroid J. 2018;7(4):167-186. doi: 10.1159/000490384

11. Румянцев П.О., Коренев С.В. История появления терапии радиоактивным йодом // Клиническая и экспериментальная тиреоидология. - 2015. - Т.11. - №4. - C. 51-55. [Rumiantsev PO, Korenev SV. The history of radioiodine therapy beginnings. Clinical and experimental thyroidology. 2015;11 (4):51-55. (In Russ.)]. doi: 10.14341/ket2015451-55.

12. Румянцев П.О., Кияев А.В. Шеремета М.С., Чикулаева О.А. Радиойодтерапия тиреотоксикоза у детей и подростков. Показания, эффективность и безопасность. Обзор литературы // Эндокринная хирургия. - 2016. - Т.10. — №4. - C. 6-12. [Rumiantsev PO, Kiyaev AV, Sheremeta MS, Chikulaeva OA. Radioiodine therapy of thyrotoxicosis in children and adolescents. Indications, efficacy and safety. Literature review. Endocrine surgery. 2016;10(4):6-12. (In Russ.)]. doi: 10.14341/serg201646-12.

13. Gambhir S, Barai S. Radioiodine for treatment of graves' disease in pediatric population: an ideal treatment. World J Endocr Surg. 2010;2(2):77-80. doi: 10.5005/jp-journals-10002-1026.
14. Ross DS, Burch HB, Cooper DS, et al. 2016 American Thyroid Association guidelines for diagnosis and management of hyperthyroidism and other causes of thyrotoxicosis. Thyroid. 2016;26(10):1343-1421. doi: 10.1089/thy.2016.0229.

15. Stokkel MP, Handkiewicz Junak D, Lassmann M, et al EANM procedure guidelines for therapy of benign thyroid disease. Eur J Nucl Med Mol Imaging. 2010;37(11):2218-2228. doi: 10.1007/s00259-010-1536-8.

16. Pandey M, Jain A. ROC Curve: Making way for correct diagnosis. SP11-PharmaSUG 2016 [Internet]. Available from: https://www. pharmasug.org/proceedings/2016/SP/PharmaSUG-2016-SP11.pdf.

17. Rivkees SA, Cornelius EA. Influence of iodine-131 dose on the outcome of hyperthyroidism in children. Pediatrics. 2003;111(4 Pt 1):745-749. doi: 10.1542/peds.111.4.745.

18. Kaplowitz PB, Jiang J, Vaidyanathan P. Radioactive iodine therapy for pediatric Graves' disease: a single-center experience over a 10-year period. J Pediatr Endocrinol Metab. 2019;33(3):383-389. doi: 10.1515/jpem-2019-0316.

19. Namwongprom S, Unachak K, Dejkhamron PM, et al. Radioactive iodine for thyrotoxicosis in childhood and adolescence: Treatment and outcomes. JCRPE J Clin Res Pediatr Endocrinol. 2013;5(2):95-97. doi: 10.4274/Jcrpe.951.

20. McCormack S, Mitchell DM, Woo M, et al. Radioactive iodine for hyperthyroidism in children and adolescents: referral rate and response to treatment. Clin Endocrinol (Oxf). 2009;71 (6):884-891. doi: 10.1111/j.1365-2265.2009.03565.x.

Рукопись получена: 12.04.2020. Одобрена к публикации: 11.09.2020. Опубликована online: 28.09.2020.

ИНФОРМАЦИЯ ОБ АВТОРАХ [AUTHORS INFO]

*Румянцев Павел Олегович, д.м.н. [Pavel O. Rumyantsev, MD, PhD]; адрес: 117036 Москва, ул. Дм. Ульянова, д. 11, к. 2 [address: 11 bld 2, Dm. Ulyanova street, 117036 Moscow, Russia]; eLibrary SPIN-код: 7085-7976; ORCID: https://orcid.org/0000-0002-7721-634X; e-mail: pavelrum@gmail.com

Саенко Владимир Александрович, к.б.н. [Vladimir A.Saenko, PhD]; eLibrary SPIN-код: 3831-0973;

ORCID: https://orcid.org/0000-0003-2844-3121; e-mail: saenko@nagasaki-u.ac.jp

Дзейтова Дали Султановна [Dali S. Dzeytova, MD]; eLibrary SPIN-код: 8761-7619;

ORCID: https://orcid.org/0000-0003-3071-4314; e-mail:dzdali@gmail.com

Трухин Алексей Андреевич [Alexey A. Trukhin]; eLibrary SPIN-код:4398-9536 ;

ORCID: https://orcid.org/0000-0001-5592-4727; e-mail: alexey.trukhin12@gmail.com

Шеремета Марина Сергеевна, к.м.н. [Marina S. Sheremeta, MD, PhD]; eLibrary SPIN-код: 7845-2194;

ORCID: https://orcid.org/0000-0003-3785-0335; e-mail: marina888@yandex.ru

Слащук Константин Юрьевич [Konstantin Y. Slashchuk, MD]; eLibrary SPIN-код: 3079-8033;

ORCID: https://orcid.org/0000-0002-3220-2438; e-mail: slashuk911@gmail.com

Дегтярев Михаил Владимирович [Mikhail V. Degtyarev, MD]; eLibrary SPIN-код: 7725-7831;

ORCID: https://orcid.org/0000-0001-5652-2607; e-mail: germed@mail.ru

Серженко Сергей Сергеевич [Sergey S. Serzhenko, MD]; eLibrary SPIN-код: 4713-8986;

ORCID: https://orcid.org/0000-0003-2326-1396; e-mail: vv1ld@yandex.ru

Ясюченя Валентина Сергеевна [Valentina S. Yasuchenia, MD]; eLibrary SPIN-код: 3810-5848;

ORCID: https://orcid.org/0000-0002-7624-7953; e-mail: loveissiberia@gmail.com

Захарова Светлана Михайловна, к.м.н. [Svetlana M.Zakharova, MD, PhD]; eLibrary SPIN-код: 9441-4035;

ORCID: https://orcid.org/0000-0001-6059-2827; e-mail: smzakharova@mail.ru

Сирота Ярослав Игоревич [Yaroslav I. Sirota]; eLibrary SPIN-код: 6691-6741;

ORCID: https://orcid.org/0000-0002-0613-9543; e-mail: yaroslawsirota@gmail.com

\section{ЦИТИРОВАТЬ:}

Румянцев П.О., Саенко В.А., Дзейтова Д.С., Трухин А.А., Шеремета М.С., Слащук К.Ю., Дегтярев М.В., Серженко С.С., Ясюченя В.С., Захарова С.М., Сирота Я.И. Предикторы эффективности терапии радиоактивным йодом болезни Грейвса у детей и подростков // Проблемы эндокринологии. — 2020. — Т. 66. — №4. — С. 68-76. doi: https://doi.org/10.14341/probl12390

\section{TO CITE THIS ARTICLE:}

Rumyantsev PO, Saenko VA, Dzeytova DS, Trukhin AA, Sheremeta MS, Degtyarev MV, Slashchuk KY, Serzhenko SS, Yasuchenia VS, Zakharova SM, Sirota YI. Predictors of the efficacy of radioiodine therapy of Graves' disease in children and adolescents. Problems of Endocrinology. 2020;66(4):68-76. doi: https://doi.org/10.14341/probl12390 